\title{
UNA EQUAZIONE DI STATO PER I SOLIDI
}

\section{P. E. VALLE}

Introduzione. - La teoria classica dei solidi è stata sviluppata principalmente da Einstein, Delye, Grüneisen e Born.

In questa teoria l'energia interna di un solido è costituita dall'energia d'interazione fra le particelle e dall'energia di agitazione termica.

Se il solido è isotropo, omogeneo, di tipo monoatomico, l'energia di agitazione termica può essere identificata con l'energia di un sistema di onde longitudinali e trasversali, la cui lunghezza però non può superare il doppio della distanza tra due particelle.

Una pressione esterna, di tipo idrostatico, che agisca sul solido, viene equilibrata dalla pressione interna dovuta allinterazione fra le particelle e dalla pressione di radiazione del sistema di onde che rappresenta l'agitazione termica. Questa ricerca costituisce uno sforzo per valutare la pressione dovuta all'interazione fra le particelle di un solido, rimanendo nel quadro della teoria classica.

Teoria. - Le frequenze massime delle onde longitudinali e trasversali relative ad un solido isotropo ed omogeneo sono date da $(1-2-3-4)$

$$
\ddot{r}_{m}=\ddot{r}_{m}\left(\frac{3 N}{4 \pi A}\right)^{1 / 3} e^{1 / 3} \quad(m=l, t)
$$

dove $v_{\mathrm{m}}$ ì la velocilì dell'onda, $N$ il numero d'Arogadro, $A$ il peso atomico e o la densitì.

Le temperature caratteristiche sono

$$
\Theta_{\mathrm{m}}=\frac{\underline{h} \varkappa_{m}}{\bar{i}} \quad(m=l, t)
$$

in cui $h$ è la costante di Planck e $k$ la costante di Boltzmann.

Nella teoria di Delye si ha una sola frequenza massima $v_{D}$, espressa da

$$
v_{\mathrm{D}}=\left(\frac{1}{v_{\mathrm{I}}^{3}}+\frac{2}{v_{\mathrm{t}}^{3}}\right)^{-1 / 3}\left(\frac{9 N}{4 \pi A}\right)^{1 / 3} e^{1 / 3}
$$


e quindi una sola temperatura caratteristica

$$
\Theta_{\mathrm{D}}=\frac{l \cdot \ddot{\mathrm{D}}_{\mathrm{D}}}{k}
$$

Si può facilmente dedurre dalle [1], [2], [3] e [4] che

$$
\frac{3}{\Theta^{3}}=\frac{1}{\Theta_{\mathrm{l}}^{3}}+\frac{2}{\Theta_{\mathrm{t}}{ }^{3}}
$$

Le energie termiche $U_{\mathrm{m}}$ hanno la forma (1-2-3-4)

$$
U_{\mathrm{u}}=\frac{R T}{A} D\left(X_{\mathrm{m}}\right) \quad(m=l, t)
$$

in cui

$$
X_{\mathrm{m}}=\frac{\Theta_{\mathrm{nu}}}{T}
$$

e $D\left(X_{u 1}\right)$ è la funzione di Debye definita da

$$
D\left(X_{\mathrm{w}}\right)=\frac{3}{X_{\mathrm{m}}^{3}} \int_{0}^{X_{\mathrm{m}}} \frac{\xi^{3} d \xi}{e^{\xi}-1}+\frac{3}{8} X_{\mathrm{w}}
$$

Le entropie $S_{\mathrm{m}}$ si possono esprimere mediante le relazioni

$$
S_{\mathrm{u}}=-\frac{R}{A} \log \left(e^{\mathrm{Xm}}-1\right)+\frac{4 R}{A X^{3}{ }_{\mathrm{m}}} \int_{\mathrm{o}}^{\mathrm{X}^{\mathrm{m}}} \frac{\xi^{3} e^{\overline{5}}}{e^{\xi}-1} d \xi(m=l, t)
$$

Se $U$ ed $S$ sono l'energia interna e l'entropia complessive, si avrà

$$
\begin{aligned}
& U=\Sigma U_{\mathrm{m}} \\
& S=\Sigma S_{\mathrm{m}}
\end{aligned} \quad(m=l, t, t)
$$

L'equazione di stato può essere scritta nella forma (1.2-5.6)

$$
p=f(\varrho)+\varrho \Sigma \gamma_{\mathrm{m}} U_{\mathrm{ut}} \quad(m=l, t, t)
$$

nella quale $p$ è la pressione, $f(\varrho)$ è la pressione interna dovuta all'interazione tra le particelle e $\gamma_{m}$ è dato da

$$
\gamma_{\mathrm{m}}=\frac{\partial \log \left(v_{\mathrm{m}} \varrho^{1 / 3}\right)}{\partial \log \varrho} \quad(m=l, t)
$$


In questa teoria le costanti elastiche, che determinano le frequenze delle onde di agitazione termica, dipendono soltanto dalle forze interatomiche. Pertanto tali costanti dovranno essere valutate in modo da non implicare variazioni delle energie di agitazione termica, e dovranno inoltre risultare indipendenti dalle energie termiche stesse.

Dato che l'equazione di stato [11] è valida soltanto per variazioni isotrope ed omogenee di volume, è necessario prendere in considerazione l'incompressibilità $K$, la quale è legata alla velocità delle onde longitudinali e trasversali dall'espressione

$$
\frac{K}{\rho}=w^{-}-\frac{4}{3} v_{\mathrm{i}}
$$

La valutazione di $K$ dovrà essere effettuata mediante la relazione

$$
K=\varrho\left(\frac{\partial p}{\partial \varrho}\right)_{U_{1}, U_{t}}
$$

e dovranno essere soddisfatte le condizioni

$$
\left(\frac{\partial K}{\partial U_{1} / \varrho, U_{\mathrm{t}}}=0 \quad, \quad\left(\frac{\partial K}{\partial U_{\mathrm{t}}}\right)_{\varrho, U_{1}}=0\right.
$$

Dall'equazione di stato, dalle [14] e [15] si ha

$$
K=\varrho \frac{d f}{a_{\varrho}}
$$

e

$$
\begin{aligned}
& \varrho \gamma_{1}=\varrho_{0} \gamma_{0} \\
& \varrho \gamma_{1}=\varrho_{0} \gamma_{o t}
\end{aligned}
$$

dove $Q_{n}, \gamma_{o l}, \gamma_{01}$ sono certi valori iniziali di queste quantità.

Sostituendo poi le espressioni di $\gamma_{m}$ date dalle [12], nelle [17] si ottiene

$$
\varrho \frac{d \lg g\left(v_{1} \varrho^{1 / 3}\right)}{a l \cdot g \varrho}=\gamma_{\mathrm{ol}} \varrho_{\mathrm{o}} \quad, \quad \varrho \frac{d \log \left(v_{\mathrm{l}} \varrho^{1 / 3}\right)}{d \log \varrho}=\gamma_{\mathrm{ol}} \varrho_{\mathrm{o}}
$$

le quali integrate forniscono subito

$$
v_{1}=v_{01}\left(\frac{\varrho^{0}}{\varphi}\right)^{1 / 3} e^{\gamma_{01}\left(1-\frac{O_{0}}{\varrho}\right)}, v_{t}=v_{n}\left(\frac{\varrho_{0}}{\varrho}\right)^{1 / 3} e^{\gamma_{01}}\left(1-\frac{\rho_{0}}{\varrho}\right)
$$


Pertanto le velocità delle onde elastiche di agitazione termica dipendono soltanto dalla distanza media tra le particelle del solido. Le relazioni [18] possono avere un certo interesse per le ricerche geofisiche.

Le frequenze massime di un solido si possono otlenere combinando le [18] con le [1].

Si avrà

$$
\gamma_{\mathrm{m}}=\because_{\mathrm{om}} \gamma_{\mathrm{ord}}\left(1-\frac{\varrho_{\mathrm{o}}}{\mathrm{e}}\right)
$$

Allo scopo di valutare la pressione interna dovuta all'interazione tra le particelle, conviene dedurre un'espressione di $K$.

Dalle equazioni [13] e [18] si ha

$$
\frac{K}{\varrho}=\left(\frac{\varrho_{\mathrm{o}}}{\varrho}\right)^{2 / 3}\left[v_{\mathrm{o} 1}^{2} e^{2 \gamma_{\mathrm{ol}}\left(1-\frac{\varrho^{\circ}}{\varrho}\right)}-\frac{4}{3} v_{\mathrm{o} 1}^{2} e^{2 \gamma_{\mathrm{ol}}\left(1-\frac{\varrho_{\mathrm{o}}}{\varrho}\right)}\right]
$$

la quale può essere scritta nella forma

$$
K=K_{\mathrm{o}} \varphi\left(\frac{\varrho}{\varrho_{\mathrm{o}}}\right)^{1 / 3} e^{2 \gamma_{\mathrm{ol}}\left(1-\frac{\varrho_{\mathrm{o}}}{\varrho}\right)}
$$

dove

$$
\begin{gathered}
K_{\mathrm{o}}=\varrho_{0}\left(v_{\mathrm{o} 1}^{\circ}-\frac{4}{3} v_{\mathrm{at}}^{2}\right) \\
\varphi=\frac{1-a^{2} e^{2\left(\gamma_{\mathrm{ot}}-\gamma_{\mathrm{ol}}\right)\left(1-\frac{\varrho_{\mathrm{o}}}{\varrho}\right)}}{1-a^{2}}
\end{gathered}
$$

e

con

$$
a^{2}=\frac{4}{3} \frac{v_{01}^{2}}{v_{01}^{2}}
$$

Poiché è da ritenere che $\mathrm{i}$ valori di $\gamma_{o l}$ e $\gamma_{o t}$ siano assai prossimi

$$
\varphi=e^{\frac{2 a^{2}}{1-a^{2}}\left(\gamma_{o l}-\gamma_{o t}\right)\left(1-\frac{\varrho_{0}}{\varrho}\right)}
$$

e quindi l'equazione [20] diviene

$$
K=K_{n}\left(\frac{\varrho}{\varrho_{0}}\right)^{1 / 3} e^{2 \Gamma\left(I-\frac{\varrho_{0}}{\varrho}\right)}
$$


dove si è posto

$$
\Gamma=\gamma_{\mathrm{ol}}+\frac{a^{2}}{1-a^{2}}\left(\gamma_{\mathrm{ol}}-\gamma_{\mathrm{ot}}\right)
$$

E possiluile ora valutare la pressione interna $f(\varrho)$.

Dalla relazione $[16]$ si ha

$$
f(\varrho)-f\left(\varrho_{0}\right)=\int_{\varrho_{0}}^{\varrho} \frac{K}{\varrho} d \varrho
$$

o anche, tenuto conto della [23],

$$
f(\varrho)-f\left(\varrho_{\mathrm{o}}\right)=K_{\mathrm{o}} \int_{1}^{\frac{\varrho}{\varrho_{\mathrm{o}}}}\left(\frac{\varrho_{\mathrm{o}}}{\varrho}\right)^{2 / 3} e^{2 \Gamma\left(1-\frac{\varrho_{\mathrm{o}}}{\varrho}\right)} d\left(\frac{\varrho}{\varrho_{\mathrm{o}}}\right)
$$

L'integrale a secondo membro della [25] può essere scritto nella forma

$$
e^{2 \Gamma}(2 \Gamma)^{1 / 3} \int_{\frac{1}{2 \Gamma}}^{\frac{1}{2 \Gamma} \xi^{-2 / 3} e^{-\frac{1}{\xi}} d \xi}
$$

e quindi la [25] diviene

$$
f(\varrho)-f\left(\varrho_{\mathrm{o}}\right)=K_{\mathrm{o}}{ }^{2}{ }^{2}(2 \Gamma)^{1 / 3}\left[F\left(\frac{1}{2 \Gamma} \frac{\varrho}{\varrho_{\mathrm{o}}}\right)-F\left(\frac{1}{2 \Gamma}\right)\right]
$$

dove

$$
F\left(\frac{1}{2 \Gamma} \frac{\varrho}{\varrho_{0}}\right)-F\left(\frac{1}{2 \Gamma}\right)=\int_{\frac{1}{2 \Gamma}}^{\frac{1}{2 \Gamma}} \frac{\varrho}{\varrho_{0}} \frac{2}{3} e^{-\frac{1}{\xi}} d \xi
$$

La forma definitiva dell'equazione di stato risulta pertanto la seguente

$$
\begin{aligned}
p-p_{\mathrm{o}} & =K_{\mathrm{o}} e^{2 \Gamma}(2 \Gamma)^{1 / 3}\left[F\left(\frac{1}{2 \Gamma} \frac{\varrho}{\varrho_{\mathrm{o}}}\right)-F\left(\frac{1}{2 \Gamma}\right)\right]+ \\
& +\varrho_{\mathrm{o}} \gamma_{\mathrm{ol}}\left(U_{\mathrm{l}}-U_{\mathrm{ol}}\right)+2 \varrho_{\mathrm{o}} \gamma_{\mathrm{ol}}\left(U_{\mathrm{t}}-U_{\mathrm{ot}}\right.
\end{aligned}
$$


nella quale l'indice zero si riferisce ad un certo stato iniziale del solido.

È utile valutare alcune grandezze termodinamiche. Il calore specifico a volume costante è dato da

$$
C_{\mathrm{v}}=\left(\frac{\partial U}{\partial T}\right)_{0}
$$

o anche

$$
C_{\mathrm{v}}=\Sigma C_{\mathrm{vm}} \quad(m=l, t, t)
$$

in cui

$$
C_{\mathrm{rm}}=\frac{3 R}{A}\left[\frac{4}{X_{\mathrm{m}}^{3}} \int_{\mathrm{o}}^{\mathrm{Xm}} \frac{\xi^{3} d \xi}{e^{\xi}-1}-\frac{X_{\mathrm{m}}}{e^{\mathrm{Xm}}-1}\right]
$$

Dall'equazione di stato si ha subito

$$
\alpha K_{\mathrm{T}}=\left(\frac{\partial p}{\partial T}\right)_{\mathrm{Q}}=\varrho_{\mathrm{o}}\left(\gamma_{\mathrm{ol}} C_{\mathrm{vl}}+2 \gamma_{\mathrm{ol}} C_{\mathrm{v} 1}\right)
$$

nella quale $K_{\mathrm{T}}$ è l'incompressibilità isoterma e $a$ la dilatazione termica.

L'espressione [32] è nota sotto il nome di "legge di Grüneisen ". Dato che l'incompressibilità isoterma è data da

$$
K_{\mathbf{T}}=\rho\left(\frac{\partial p}{\partial \rho / T}\right.
$$

dall'equazione di stato consegue

$$
K_{\mathrm{T}}=K+\varrho_{\mathrm{o}} \varrho \Sigma \gamma_{\mathrm{ox}}\left(\frac{\partial U_{\mathrm{m}}}{\partial \varrho}\right)_{\mathrm{T}} \quad(m=l, t, t)
$$

che, con qualche passaggio, si può porre nella forma

$$
K_{\mathrm{T}}=K+\varrho\left(\frac{\varrho_{0}}{\varrho}\right)^{2} \sum_{\mathrm{om}_{\mathrm{m}}} T\left(\frac{R}{A} D\left(\vee_{\mathrm{m}}\right)-C_{\mathrm{vn}}\right) \quad(m=l, t, t)
$$

Se la temperatura $T$ è maggiore delle temperature caratteristiche, allora è praticamente

$$
\frac{R}{A} D\left(X_{\mathrm{m}}\right)-C_{\mathrm{vm}}=0
$$

e quindi

$$
K_{\mathrm{T}}=K
$$


Le espressioni [23], [28] e [32] divengono in questo caso

$$
\begin{gathered}
K_{\mathrm{T}}=K_{\mathrm{oT}}\left(\frac{\varrho}{\varrho_{\mathrm{o}}}\right)^{1 / 3} e^{2 \Gamma\left(1-\frac{\varrho_{\mathrm{o}}}{\varrho}\right)} \\
p-p_{\mathrm{o}}=K_{\mathrm{oT}} e^{2 \Gamma}(2 \Gamma)^{1 / 3}\left[F\left(\frac{1}{2 \Gamma} \frac{\varrho}{\varrho_{\mathrm{o}}}\right)-F\left(\frac{1}{2 \Gamma}\right)\right]+ \\
+\frac{3 \varrho_{\mathrm{o}} \gamma_{\mathrm{o}} R}{A}\left(T-T_{\mathrm{o}}\right) \\
u K_{\mathrm{I}}=\frac{3 \varrho_{\mathrm{o}} \gamma_{\mathrm{o}} R}{A}
\end{gathered}
$$

dove

$$
\gamma_{\mathrm{o}}=\frac{\gamma_{\mathrm{ol}}+2 \gamma_{\mathrm{ot}}}{3}
$$

Quindi, se la temperatura è alıa, le velocità $v_{\mathrm{m}}$ delle onde elastiche si identificano con le velocità isoterme. Dato poi che la rigidità adiabatica è uguale alla rigidità isoterma, la velocità delle onde trasversali $v_{t}$ coincide anche con la velocità adiabatica. Se invece la temperatura è inferiore alle temperature caratteristiche, la quantità

$$
\sum \gamma_{\mathrm{om}}^{2} T\left(\frac{R}{A} D\left(X_{\mathrm{m}}\right)-C_{\mathrm{vm}}\right)
$$

cresce al crescere di $X_{\mathrm{m}}$. Per $X_{\mathrm{n}} \rightarrow \infty$ si ha

$$
K_{\mathrm{T}}=K+\frac{3}{8} \frac{R}{A} \varrho\left(\frac{\varrho_{\mathrm{o}}}{\varrho}\right)^{2} \Sigma \gamma_{\mathrm{om}}^{2} \Theta_{\mathrm{m}} \quad(m=l, t, t)
$$

Il secondo termine a secondo membro della [38] è dovuto all'agitazione termica delle particelle allo zero assoluto, ma in generale sarà piuttosto piccolo.

Dalla teoria sviluppata in questo paragrafo, si possono dedurre diverse relazioni termodinamiche. $\mathrm{Si}$ ha per es. ad alte temperature

$$
\frac{1}{\alpha \beta_{\mathrm{T}}}\left(\frac{\partial \beta_{\mathrm{T}}}{\partial T}\right)_{\mathrm{P}}=\frac{1}{\alpha^{2}}\left(\frac{\partial \alpha}{\partial T}\right)_{\mathrm{P}}=\left(\frac{\partial K_{\mathrm{T}}}{\partial P}\right)_{\mathrm{T}}
$$

in cui

$$
\left(\frac{\partial K_{\mathrm{T}}}{\partial p}\right)_{\mathrm{T}}=\frac{1}{3}+2 \Gamma \frac{\varrho_{\mathrm{o}}}{\varrho}
$$

e $\beta_{\mathrm{T}}$ rappresenta la compressibilità isoterma. 
Confronto con le misure di compressione. - Le misure di compressione effettuate da Bridgman $\left({ }^{s}\right)$, consentono un confronto della teoria con $\mathrm{i}$ risultati sperimentali.

A questo scopo sono stati presi in considerazione $i$ metalli alcalini litio, sodio e potassio, i quali posseggono un'alta compressibilità

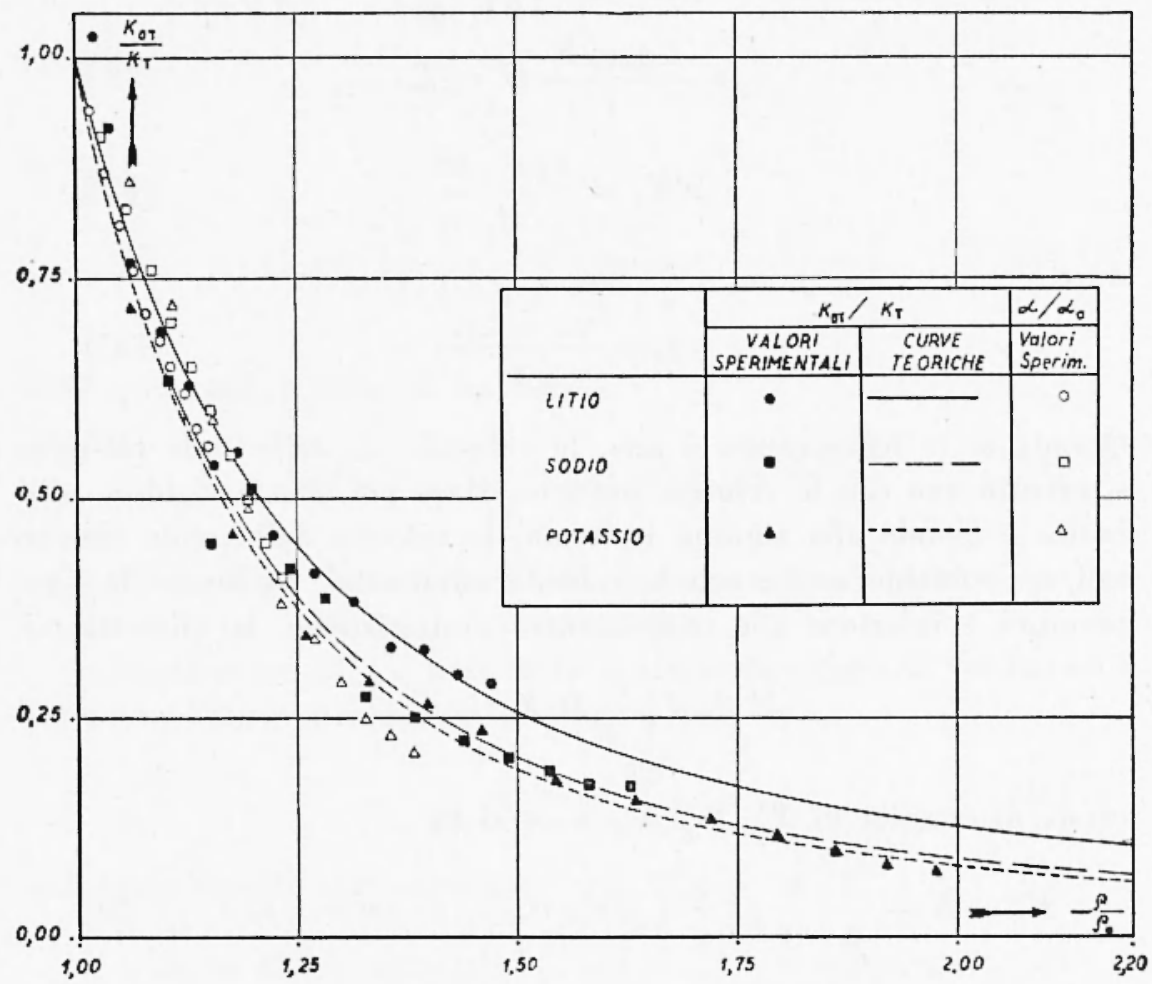

Fig. 1 - Compressibilità isoterma e dilatazione termica dei metalli alcalini.

e non presentano transizioni polimorfiche almeno fino alla pressione di $100.000 \mathrm{Kg} / \mathrm{cm}^{2}$.

Le misure effettuate da Bridgman fino a $100.000 \mathrm{Kg} / \mathrm{cm}^{2}$, partono da $25.000 \mathrm{Kg} / \mathrm{cm}^{2}$. Nella tabella $I$ sono contenuti $\mathrm{i}$ volumi relativi del litio, sodio e potassio da 30.000 a $100.000 \mathrm{Kg} / \mathrm{cm}^{2}$ e gli analoghi volumi da zero a $30.000 \mathrm{Kg} / \mathrm{cm}^{2}$, ottenuti da Bridgman con un differente dispositivo sperimentale. I due gruppi di misure si raccordano in modo abbastanza soddisfacente. I dati contenuti nella tabella 1 si riferiscono alla temperatura ambiente. 
In condizioni isoterme, trascurando i termini che dipendono dalle energie termiche, l'equazione di stato si riduce a

$$
p=K_{\mathrm{o} \cdot \mathrm{r}} e^{2 \Gamma}(2 \Gamma)^{1 / 3}\left[F\left(\frac{1}{2 \Gamma} \frac{\varrho}{\varrho_{0}}\right)-F\left(\frac{1}{2 \Gamma}\right)\right]
$$

e l'incompressibilità isoterma d̀ data da

$$
K_{\mathrm{I}}=K_{\mathrm{oT}}\left(\frac{\varrho}{\varrho_{\mathrm{o}}}\right)^{1 / 3} e^{2 \Gamma\left(1-\frac{\varrho_{\mathrm{o}}}{\varrho}\right)}
$$

dove l'indice zero si riferisce allo zero della pressione e alla temperatura ambiente.

Per effettuare il confronto della equazione [42] con i dati sperimentali è necessario valutare $K_{\text {ot }}$ e $2 \Gamma^{*}$. I valori medi di $K_{\Gamma}$ possono essere dedotti dai dati contenuti nella tabella 1 .

\section{TaBella 1}

Volumi relativi dei metalli alcalini $L i, N a, K$ a temperatura ambiente fino a $100.000 \mathrm{Kg} / \mathrm{cm}^{2}$

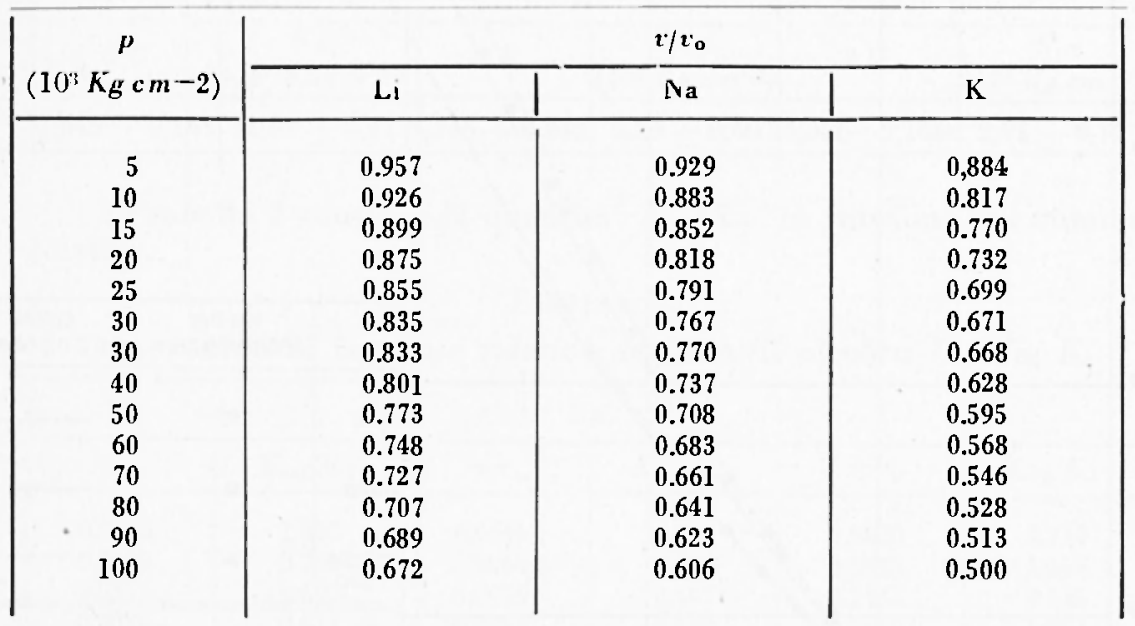

* Una stima approssimata della temperatura di Debye, alla temperatura am. biente e alla pressione zero, ha fornitn per il litio $\Theta_{D}=430^{\circ} \mathrm{K}$, per il sodio $\Theta_{D}=190^{\circ} K$ e per il potassio $\Theta_{D}=110^{\circ} K$. Pertanto $i$ dati sperimentali del litio si riferiscono ad una temperatura inferiore alla temperatura di Debye.

Le temperature di Debye del sodio e del potassio si avvicinano alla temperatura ambiente verso i $100.000 \mathrm{Kg} / \mathrm{cm}^{2}$. 


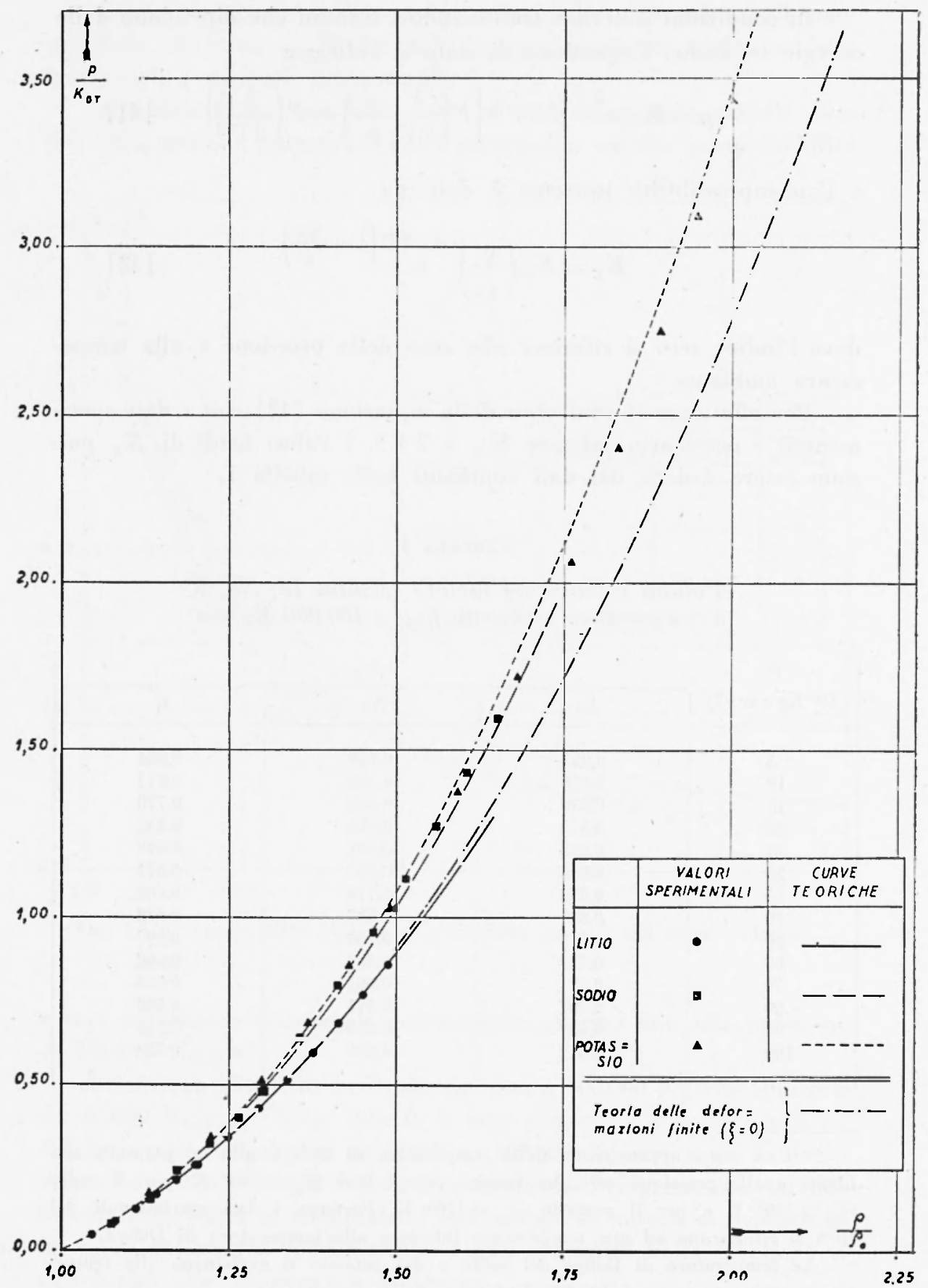

Fig. 2 - Isoterme dei metalli alcalini fino a $100.000 \mathrm{~kg} / \mathrm{cm}^{2}$ a temperatura ambiente. 
Si ha

dove

$$
K_{\mathrm{T}}\left(\frac{\varrho_{\mathrm{o}}}{\varrho}\right)=-\frac{v}{v_{\mathrm{o}}} \frac{\Delta p}{\frac{v_{2}}{v_{\mathrm{o}}}-\frac{v_{1}}{v_{\mathrm{o}}}}
$$

$$
\frac{\varrho_{0}}{\varrho}=\frac{v}{v_{0}}=\frac{1}{2}\left(\frac{v_{1}}{v_{0}}+\frac{v_{2}}{v_{0}}\right)
$$

L'equazione [42] può essere anche scritta nella forma

$$
\log K_{\mathrm{T}}+\frac{1}{3} \log \frac{\mathrm{t}}{\varrho}=\log K_{\mathrm{Tu}}+\left(1-\frac{\varrho_{\mathrm{o}}}{\varrho}\right) 2 \Gamma
$$

Sostituendo in questa relazione i valori numerici di $K_{\mathrm{T}}$ e $\varrho_{\mathrm{o}} / \varrho$ ottenuti dalla tabella 1 mediante le [43], si ottiene un sistema di equazioni per determinare $K_{\text {or }}$ e $\Gamma$. Tale sistema di equazioni è stato isollo col metodo dei minimi quadrati. I risultati del calcolo sono contenuti nella tajuella 2 .

\section{Tabella 2}

\begin{tabular}{|c|c|c|c|c|c|}
\hline \multicolumn{2}{|c|}{$\mathrm{L}, \mathrm{i}$} & \multicolumn{2}{|c|}{ N. } & \multicolumn{2}{|c|}{$\mathrm{K}$} \\
\hline $2 \mathrm{I}$ & $\begin{array}{c}K_{\mathrm{oT}} \\
\left(10^{1} \mathrm{Kg} \mathrm{cm}^{-2}\right)\end{array}$ & $2 \mathrm{I}$ & $\begin{array}{c}\overline{K_{\mathrm{oT}}} \\
\left(10^{1} K_{g} \mathrm{~cm}^{-2}\right)\end{array}$ & $2 \mathrm{I}^{\prime}$ & $\begin{array}{c}K_{\mathrm{oT}} \\
\left(10^{1} K_{\mathrm{g} \mathrm{cm}-2}\right)\end{array}$ \\
\hline $3,645-0,155$ & $11,67 \pm 0,37$ & $4,345+0,242$ & $6,26 \pm 0,40$ & $4,526+0,166$ & $2,91 \div 0,17$ \\
\hline
\end{tabular}

Incompressibilità isoterma iniziale e parametro $2 \mathrm{\Gamma}$ per $i$ metalli alcalini $L i, N a, K$.

La tabella 3 contiene la quantità $K_{\mathrm{oT}} / K_{\mathrm{T}}$ in funzione del volume relativo.

\begin{tabular}{|c|c|c|c|c|c|}
\hline \multicolumn{2}{|c|}{$1, i$} & \multicolumn{2}{|c|}{$\mathrm{Na}$} & \multicolumn{2}{|c|}{$\mathbf{K}$} \\
\hline$v / 10$ & $K_{\mathrm{oT}} K_{\mathrm{T}}$ & $v / t_{0}$ & $K_{\mathrm{oT}} K_{\mathrm{T}}$ & $v v_{0}$ & $\bar{K}_{\text {oT }} K_{\mathrm{T}}$ \\
\hline 0.9785 & 1.025 & 0.9645 & 0.922 & 0.9420 & 0.717 \\
\hline 0.9415 & 0.769 & 0.9060 & 0.636 & 0.8505 & 0.459 \\
\hline 0.9125 & $0.690_{3}$ & 0.8675 & 0.447 & 0.7935 & 0.345 \\
\hline 0.8870 & 0.631 & 0.8350 & 0.510 & 0.7510 & $0.294 \mathrm{~s}$ \\
\hline 0.8650 & 0.540 & 0.8045 & 0.420 & 0.7155 & 0.268 \\
\hline 0.8450 & 0.553 & 0.7790 & 0.386 & 0.6850 & 0.238 \\
\hline 0.8170 & 0.457 & 0.7535 & 0.274 & 0.6480 & 0.180 \\
\hline 0.7870 & 0.415 & 0.7225 & 0.251 & 0.6115 & 0.157 \\
\hline 0.7605 & 0.384 & 0.6955 & 0.225 & 0.5815 & 0.135 \\
\hline 0.7375 & 0.332 & 0.6720 & 0.205 & 0.5570 & 0.115 \\
\hline 0.7170 & $0.325_{5}$ & 0.6510 & 0.192 & 0.5370 & 0.098 \\
\hline 0.6980 & 0.301 & 0.6320 & 0.178 & 0.5205 & 0.084 \\
\hline 0.6805 & $0.29 l_{s}$ & 0.6145 & 0.173 & 0.5065 & 0.075 \\
\hline
\end{tabular}

\section{Tabella 3}

Compressibilità isoterme relative dei metalli alcalini $\mathrm{Li}, \mathrm{Na}, \mathrm{K}$. 
11 confronto tra le curve teoriche di $K_{\mathrm{oT}} / K_{\mathrm{T}}$ in funzione di $\mathrm{g} / \mathrm{o}_{\mathrm{o}}$, calcolate mediante la [42] con i valori $\Gamma$ della tabella 2 , e i valori sperimentali è mostrato nella fig. 1 .

Questa figura contiene anche i valori di $\alpha / t_{0}$, che Birch $\left({ }^{7}\right)$ ha ricavato dalle misure effettuate da Bridgman tra $0^{\circ} \mathrm{C}$ e $95^{\circ} \mathrm{C}\left({ }^{10}\right)$.

Tali valori sono elencati nella tabella 4 .

\section{Tabella 4}

Effetto della pressione sulla dilatazione termica.

\begin{tabular}{|c|c|c|c|c|c|c|}
\hline \multirow{2}{*}{$\begin{array}{c}\mathrm{p} \\
\left(10^{3} K_{g} \mathrm{~cm}^{-2}\right) \\
\end{array}$} & \multicolumn{2}{|c|}{$\mathbf{L i}$} & \multicolumn{2}{|c|}{$\mathrm{Na}$} & \multicolumn{2}{|c|}{$\mathbf{K}$} \\
\hline & $v_{v} v_{0}$ & $\alpha_{i} \alpha_{0}$ & $r_{0}$ & $\alpha^{\prime} \alpha_{0}$ & $v_{i} v_{\mathrm{o}}$ & $\alpha / \alpha_{0}$ \\
\hline 2 & 0.9836 & 0.94 & 0.9705 & 0.91 & 0.9429 & 0.86 \\
\hline 4 & 0.9680 & 0.87 & $0.9+118$ & 0.83 & 0.8998 & 0.72 \\
\hline 6 & 0.9534 & 0.81 & 0.9221 & 0.76 & 0.8653 & 0.59 \\
\hline 8 & 0.9394 & 0.76 & 0.9019 & 0.70 & 0.8360 & 0.49 \\
\hline 10 & 0.9261 & 0.71 & 0.8835 & 0.65 & 0.8110 & 0.38 \\
\hline 12 & 0.9134 & 0.68 & 0.8668 & 0.60 & 0.7892 & 0.3 .1 \\
\hline 14 & 0.9016 & 0.65 & 0.8512 & 0.55 & 0.7700 & 0.29 \\
\hline 16 & 0.8906 & 0.62 & 0.8368 & 0.50 & 0.7528 & 0.25 \\
\hline 18 & 0.8802 & 0.58 & 0.8233 & 0.45 & 0.7374 & 0.23 \\
\hline 20 & 0.8704 & 0.56 & 0.8106 & 0.41 & 0,7233 & 0.21 \\
\hline
\end{tabular}

Allo scopo di confrontare l'equazione di stato [4l] con i dati della tabella 1 , è stata calcolata l'espressione

$$
F\left(\frac{1}{2 \Gamma} \frac{\varrho}{\varrho_{0}}\right)-F\left(\frac{1}{2 \Gamma}\right)
$$

Le curve teoriche di $p / K_{\mathrm{oT}}$ in funzione di $\varrho / \varrho_{\mathrm{o}}$, sono contenute nella fig. 2 , in cui sono stati riportati anche $i$ valori sperimentali.

A titolo di confronto la fig. 2 contiene inoltre la curva teorica di $p: K_{\text {or }}$, quale risulta dalla teoria delle deformazioni finite di Murnaghan. Da questa teoria si ha

$$
p / K_{\text {oT }}=\frac{3}{2}\left\{\left(\frac{\varrho}{\varrho_{0}}\right)^{\gamma / 3}-\left(\frac{\varrho}{\varrho_{0}}\right)^{5 / 3}\right\}\left\{1-\xi\left[\left(\frac{\varrho}{\varrho_{0}}\right)^{2 / 3}-1\right]+\ldots\right\}
$$

nella quale, per i metalli alcalini, Birch assume $\xi=0$.

Confronto con $i$ dati sismici. - Tra la profondità di circa $900 \mathrm{Km}$ e $2900 \mathrm{Km}$, il mantello della Terra risulta sostanzialmente uniforme e ad una temperatura superiore alla temperatura di Debye, che si aggira intorno ai $1000^{\circ} \mathrm{C}$. 
Ora ad una temperatura dell'ordine di circa $1000^{\circ} \mathrm{C}$, i silicati si comportano come semplici cristalli ionici $\left({ }^{7}\right)$, quindi può essere usata la relazione

$$
v_{\mathrm{t}}=v_{\mathrm{o}}\left(\frac{\varepsilon_{\mathrm{o}}}{\varrho}\right)^{1 / 3} e^{\nu_{\mathrm{o}}+\left(1-\frac{\varrho_{0}}{\rho}\right)}
$$

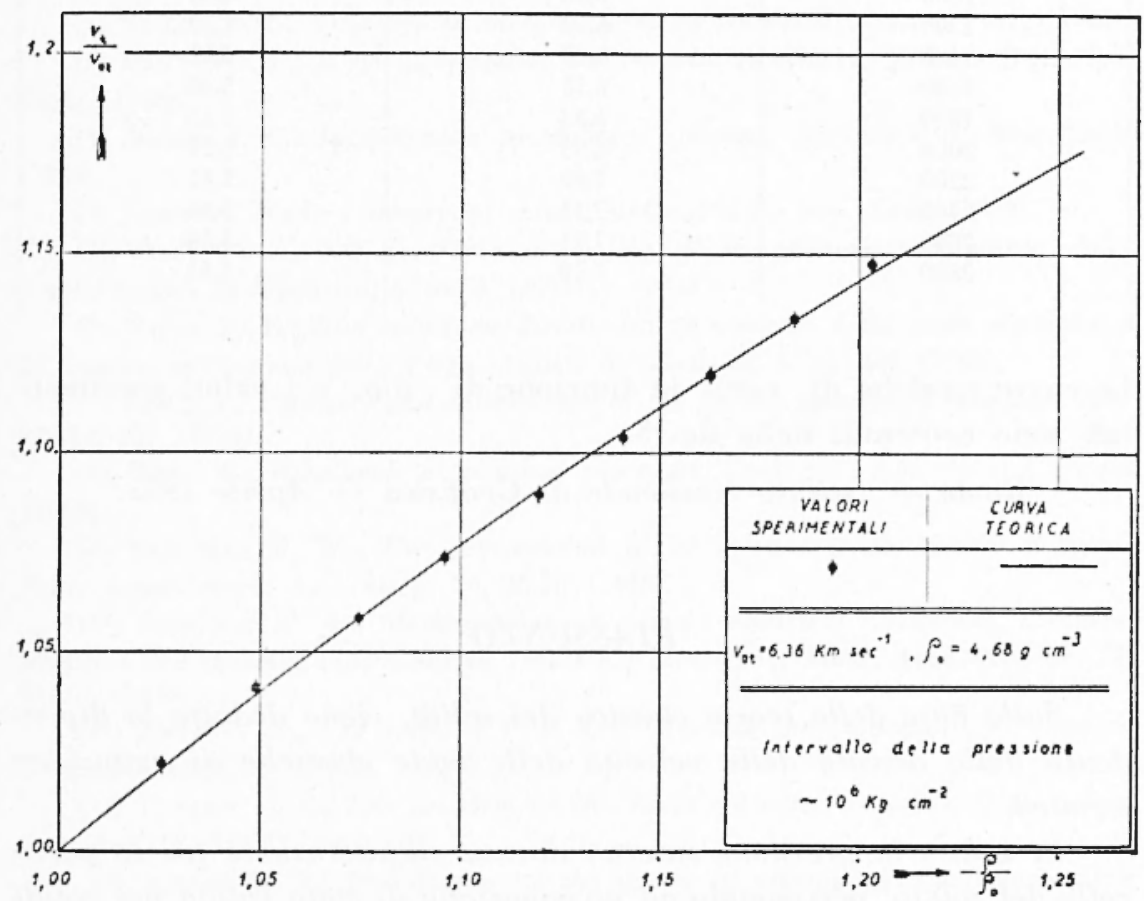

Fig. 3 - Velocità delle onde sismiche $\mathrm{S}$ nello strato $\mathrm{D}$ del mantello della Terra, in funzione della densità.

Dato che la velocità adiabatica delle onde trasversalì è identica alla velocità isoterma, è corretto confrontare la [44] con la velocità delle onde sismiche di tipo $S$.

Nella tabella 5 sono contenute le velocità delle onde $S$ ottenute da Jeffreys (11) e le densità calcolate da Bullen, in funzione della profondità (12-13). Mediante la relazione [44] e con i dati della tabella 5 , è stato calcolato il valore più probabile di Yor. E risultato

$$
\gamma_{o t}=1,169 \pm 0,004
$$




\section{Tabella 5}

Velocità delle onde $\mathrm{S}$ e densità nello strato $\mathrm{D}$ del mantello della Terra.

\begin{tabular}{|c|c|c|}
$\begin{array}{c}\mathrm{h} \\
\text { (profondità } K \boldsymbol{K})\end{array}$ & $\begin{array}{c}\boldsymbol{v}_{\mathbf{t}} \\
\left(\mathrm{km} \mathrm{sec} \mathbf{~}^{\mathbf{2}}\right)\end{array}$ \\
\hline 1000 & 6,36 & 4,68 \\
1200 & 6,50 & 4,80 \\
1400 & 6,62 & 4,91 \\
1600 & 6,73 & 5,03 \\
1800 & 6,83 & 5,13 \\
2000 & 6,93 & 5,24 \\
2200 & 7,02 & 5,34 \\
2400 & 7,12 & 5,44 \\
2600 & 7,21 & 5,54 \\
2800 & 7,30 & 5,63
\end{tabular}

Le curve teoriche di $v_{\mathrm{t}} / v_{0}$ in funzione di $\mathrm{\varrho} / \mathrm{g}_{\mathrm{o}} \mathrm{e} \mathrm{i}$ valori sperimentali sono contenuti nella fig. 3 .

$$
\text { Roma - Istituto Nazionale di Geofisica - Aprile } 1953 .
$$

\section{RIASSUNTO}

Sulla base della teoria classica dei solidi, viene dedotta la dipendenza dalla densità della velocità delle onde elastiche di agitazione termica.

Si valuta la pressione interna dovuta all'interazione fra le particelle del solido, pervenendo ad un'equazione di stato valida per solidi isotropi di tipo monoatomico o per cristalli ionici.

L'accordo della teoria con le misure di compressione effettuate da Bridgman e con $i$ dati sismici, risulta verificato entro $i$ limiti degli errori sperimentali.

\section{SUMMARY}

The velocity of elastic waves as a function of density is derived on the basis of the classical theory of solids.

The internal pressure due to the interactions of the particles of a solid is evaluated and an equation of state is deduced. 
The agreement of the theory with Bridgman's compression measurements and with the seismological data appears to be within the experimental errors.

\section{BIBLIOGR AFIA}

(1) Brit.ouin L., Tensęurs en mécanique et en elasticité. Masson, Paris (1946).

(-) Brit.oun L., Wave propagation in periodic structures. McGraw-Hill, New York (19:46).

(3) Su.ıtra J. C., Introduction to shemical physics. MeGraw-Hill, New York (1939).

(4) Stitz F, Modern theory of solids. MrGraw-Hill, New York (19.18).

(5) Valte: P. E., Sul gradiente adinbutico di temperatura nell'interno della Terra. Annali di Geofisica, 5, 41-53 (1952).

(6) Vat.t. P. E., Una relazione dircttı fra la velocità delle onde elastiche e la densitì nell'interno della Terra. Annali di Geofisica, 5, 417431 (1952).

(7) Bınch F., Elasticity and constitution of the Earth's interior. J. Geoplys. Res. $57,227-286(1952)$.

(8) Buвсн F., Handlbook of phrsical constants. Geol. Soc. Am. Special papers (1950).

(9) Bumcias P. W., The compression of 39 substances to $100.000 \mathrm{Kg} / \mathrm{cm}^{2}$. Proc. Amer. Acad. Aris Scien. 76, 55-70 (19.48).

(10) Bum,ina P. W. Measurements of certain electrical resistences, compressibilities and thermal expansions to $20.000 \mathrm{Kg} / \mathrm{cm}^{2}$. Proc. Amer. Acad. Arts Sc. 70. 71-101 (1935).

(11) JEwritys H., The time of $\mathrm{P}, \mathrm{S}$ and SKS and the velocities of $\mathrm{P}$ and $\mathrm{S}$. Geophys. Suppl. 1, 198.533 (1939).

(12) But.ex K. E., The problem of the Earth's density variation. Bull. Seism. Soc. Am. 32. 19-29 (19:12).

(13) ButLex K. E., Introduction to the theory of seismology. Cambridge, University Press (1917). 